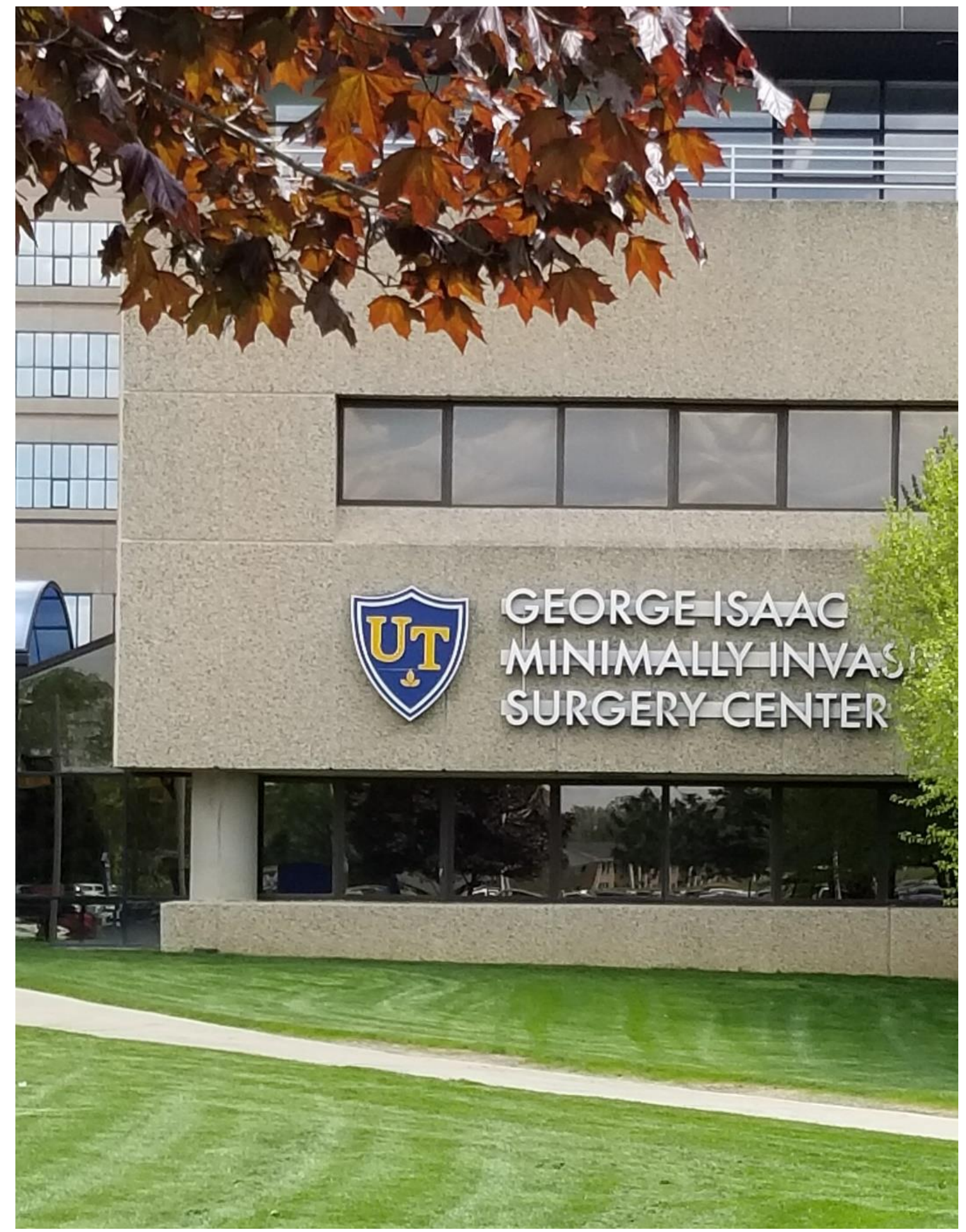




\title{
Functional castration from blunt-force trauma to motorcycle gas tank from a car versus motorcycle MVA
}

\author{
Margaret Reilly, ${ }^{a, 1}$ Damian Garcher, ${ }^{a}$ Eric Pizza ${ }^{b}$, Puneet Sindhwani ${ }^{a}$
}

${ }^{a}$ Department of Family Medicine, Health Science Campus, The University of Toledo, 3000 Arlington, Toledo OH 43614-2598, USA, and ${ }^{b}$ ProMedica Genitourinary Surgeons, Toledo, $\mathrm{OH}$.

\begin{abstract}
We present a case of bilateral testicular rupture with significant extrusion and seminiferous tubule loss in an otherwise healthy 26-year-old male as the result of a car-versus-motorcycle accident. The patient was the lone driver of a motorcycle that was T-boned, resulting in significant scrotal trauma necessitating operative intervention. Because of this, he developed subsequent severe hypogonadism, which improved with exogenous testosterone administration. We undertook a literature review, which revealed that blunt trauma is an extremely uncommon cause of acquired hypogonadism.
\end{abstract}

\section{testicular trauma | testicular rupture | acquired hypogonadism |}

A 26-year-old male without past urologic history presented to the emergency department (ED) as a trauma alert as the result of a car-versus-motorcycle accident. The patient was a helmeted driver of a motorcycle traveling at an unknown speed down a city street that T-boned a motor vehicle. The motorcyclist sustained a "fuel tank" injury from contact with his motorcycle while being ejected from the motorcycle over the motor vehicle, and traveled approximately 20 feet prior to landing on the road. As a result, he sustained multiple blunt-force injuries including full thickness lacerations. Urology was consulted in the trauma bay for further evaluation of the scrotal trauma. On exam, significant, actively bleeding avulsions of the anterior scrotum were noted bilaterally, and tunica albuginea of both testicles was clearly visualized on exam. The open wounds required urgent surgical exploration. Figures 1, 2, and 3 show the severe extent of injuries to the patient's right testicle.

On surgical exploration, we noted bilateral, nearly circumferential hemispherical testicular ruptures with significant extrusion of seminiferous tubules. The extruded seminiferous tubules were lightly debrided, and tunical defects were suture-closed in the operating room (OR) in a running fashion using 3-0 vicryl. Figures 4 and 5 show the finished product of suture closure of the left testicle. Non-viable scrotal tissue was further debrided, and the wound was copiously irrigated. The testicles were then placed back in anatomic position in each hemi-scrotum, and sub-dartos $1 / 2$ inch Penrose drains were placed bilaterally. The skin was then suture closed with a running 3-0 chromic suture. The patient recovered from his urologic injuries uneventfully after an extended hospital stay.

At his first follow-up appointment one-month post-accident, free- and total-testosterone were noted to be $0.34 \mathrm{ng} / \mathrm{dL}$ and 8.6 $\mathrm{ng} / \mathrm{dL}$, respectively. Accepted normal values of total testosterone range from $280-800 \mathrm{ng} / \mathrm{dL}$. It has been suggested that free testosterone levels below 50-65 pg/mL imply hypogonadism (1).

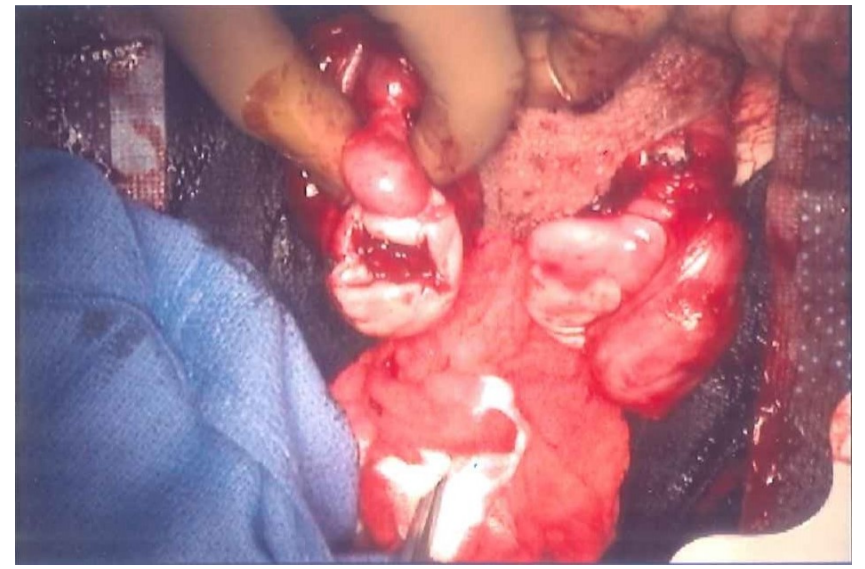

Figure 1. An almost completely hemispheric, circumferential testicular rupture is visualized at the mid-pole right testicle on intraoperative inspection. Note that the left testicular injury has already been repaired.

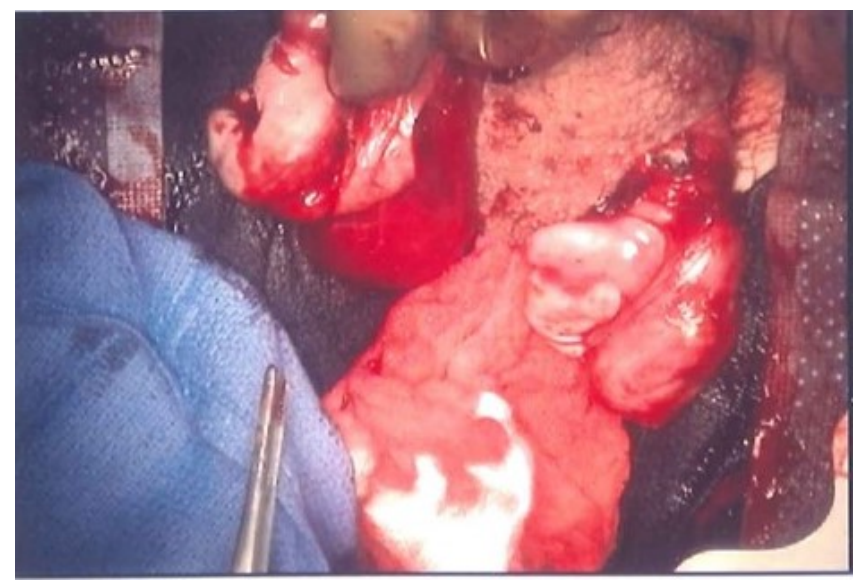

Figure 2. A medial view of the extent of the right testicular injury.

All authors contributed to this paper. ${ }^{1}$ To whom correspondence should be sent: Damian.Garcher@utoledo.edu

The authors declare no conflict of interest. Submitted: February/02/2019, published: June/13/2019.

Freely available online through the UTJMS open access option 
The patient noted fatigue, however interestingly reported normal erections/libido.

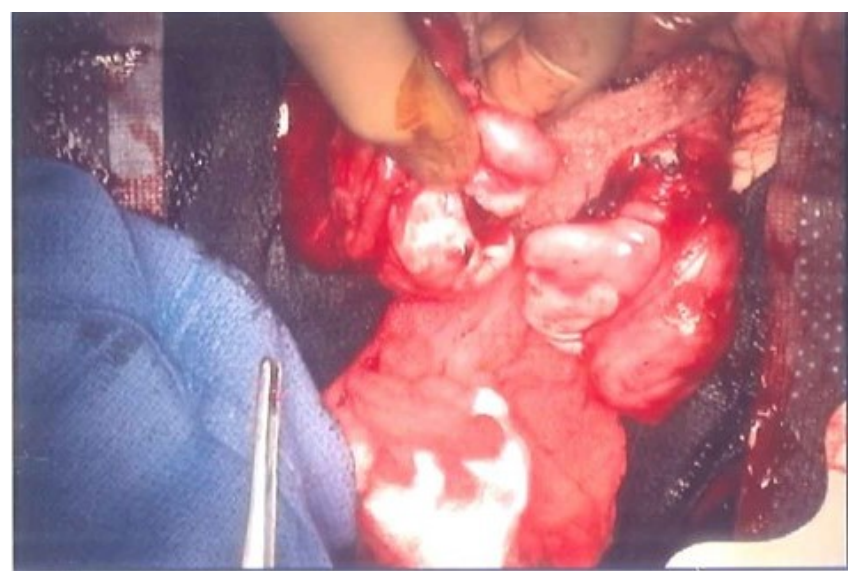

Figure 3. A lateral view of the extent of the right testicular injury.

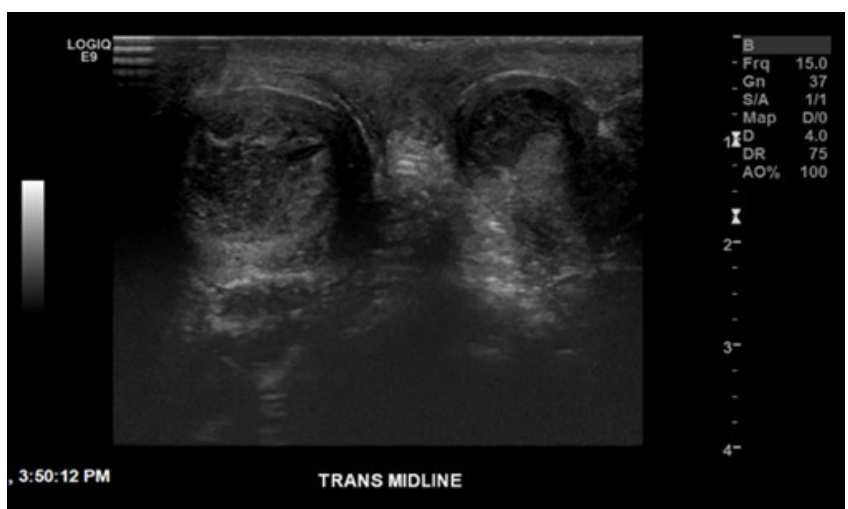

Figure 4. Cross-sectional scrotal ultrasound done approximately one month post-operatively demonstrates decreased volume of both testicles.

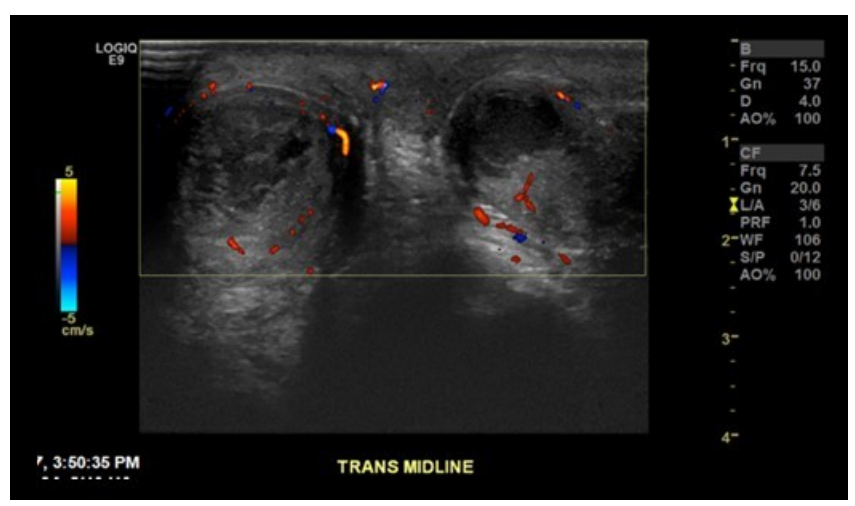

Figure 5. Scrotal ultrasound with color Doppler done approximately one month post-operatively demonstrates normal flow to both testicles.
Scrotal ultrasound at approximately one and a half months postinjury showed decreased volume with preserved blood flow, as seen in Figures 4 and 5. After declining sperm banking, he was started on $200 \mathrm{mg}$ Depo-testosterone shots every 2 weeks with subsequent normalization of testosterone levels and improvement in energy levels.

Discussion. First reported in 1818 as a crush injury from a wagon wheel, testicular trauma is now well-described in modern literature (2). Injuries range in severity from contusions to more serious entities like fractures, ruptures, dislocations, and avulsions. In one report, $28 \%$ of testicular injuries from trauma also presented with associated perineal soft tissue injuries. Although in general a rare entity in blunt trauma situations, testicular trauma is often associated with motorcycle collisions $(3,4)$. These are usually assumed to be from contact with the fuel tank, which strikes the groin and results in damage to the scrotum and perineum, commonly called a fuel tank injury $(3,5)$. Fuel tank injuries are useful in post-mortem investigations as they are usually found in the motorcycle driver and not in the passenger. It has been noted that even then these injuries are rare, as they usually result from a head on collision (5).

Bilateral testicular involvement is relatively rare, with a crude incidence rate of $6.47 \%(9 / 139$ cases) $(6,7)$. In a study of 86 patients with testicular injury, only $5(5.8 \%)$ were found to have bilateral injury (7). Penetrating trauma has been shown to be more likely than blunt trauma to produce bilateral injuries, occurring in $1.4 \%$ of cases with blunt trauma compared to $29 \%$ of those with penetrating trauma. Three men in the investigation had bilateral testicular ruptures, two of whom were treated with bilateral partial orchiectomy and one treated with orchiectomy with contralateral debridement and suturing. Testicular rupture occurs when the fibrous envelope around the testes, the tunica albuginea, is disrupted or extrusion of the seminiferous tubules occurs4. This can be detected on ultrasound, or as in our case, upon visual inspection (4). Our patient with blunt testicular trauma and significant scrotal abrasions had bilateral testicular ruptures with visible tunica albuginea. Our literature search did not yield any instances of bilateral scrotal trauma with abrasions significant enough for tunica albuginea to be clearly visible on exam.

Prior to 1972, blunt trauma to the testicles was treated with initial conservative management, after which early surgical management was established as a superior approach (7). One review found that initial conservative management led to loss of the entire testicle in $21 \%$ of cases compared to $6 \%$ in early surgery. Orchiectomy was performed in $45.5 \%$ of cases with delayed surgical exploration. The surgical management of our patient was indicated by the active scrotal bleeding and visible tunica albuginea. Surgical debridement requires a discerning eye, as it should be conservative enough to preserve viable tissue, yet adequate enough to remove dead tissue.

Testicular trauma can have detrimental effects on testicular function. Consequences range from castration, leading to hypogonadism, or rupture, exposing spermatic antigens to the immune system. Both hypogonadism and the production of anti-sperm antibodies can lead to infertility (4). In animal models, unilateral blunt testicular trauma with intact tunica albuginea was shown to affect bilateral germ cell maturation and change the sex hormone profile (8). Reperfusion of ischemic testis is thought to activate a cytokinestress-related kinase pathway, possibly causing aspermatogenesis due to germ cell-specific apoptosis. Additionally, in humans, the initial trauma may be enough to cause testicular atrophy regardless of successful repair and salvage efforts, as shown in a study by Cross et al (9). 
Reports of testicular function after trauma in the literature are sparse. In one case of bilateral testicular injury from blunt trauma with known follow-up, the patient had decreased erections with unknown testosterone after reconstruction in the OR (5). There has been one report of returned spermatogenesis after reduction and orchiopexy in a male who had experienced testicular dislocation 13 years prior (2). Another study by Redmond et al investigated testicular function after blunt testicular trauma and found evidence of testicular atrophy in four cases with known three month follow-up. Our patient with significant bilateral trauma reported normal libido and erections but had severe hypogonadism. It is difficult to know what to expect after testicular injury due to lack of follow-up, which

1. Dandona P, Rosenberg MT (2010) A practical guide to male hypogonadism in the primary care setting. Int $J$ Clin Pract 64(6):682-96.

2. Ezra N, Afari A, Wong $J$ (2009) Pelvic and scrotal trauma: CT and triage of patients. Abdominal Imaging, 34(4):541-544. doi:10.1007/s00261-008-9417-3.

3. Carvalho NMN de, Marques ACX, Souza IT de, et al (May 2018) Bilateral Traumatic Testicular Dislocation. Case Reports in Urology, 1-5. doi:10.1155/2018/7162351.

4. Addas F, Yan S, Hadjipavlou M, et al (November 2018) Testicular Rupture or Testicular Fracture? A Case Report and Literature Review. Case Reports in Urology, 1-3. doi:10.1155/2018/1323780.

5. Ihama Y, Fuke C, Miyazaki T (September 2007) A Two-Rider Motorcycle Accident Involving Injuries around Groin Area in Both the Driver and the Passenger. Legal Medicine, 9(5): 274-277. doi:10.1016/j.legalmed.2007.03.003.

6. Altarac S (1994) Management of 53 Cases of Testicular Trauma. European Urology, 25(2): 119\{123. doi:10.1159/000475264. translates to uncertainty. Our literature review failed to yield examples of testicular function after bilateral blunt testicular trauma with rupture of tunica albuginea. Reports like ours can aid in counselling patients after traumatic testicular injury, but increased follow-up is needed to accurately predict functional status.

Conclusion. Blunt force trauma from motorcycle MVA may potentially cause hypogonadism in severe cases. A literature review demonstrated that this is an extremely uncommon cause of severe testicular injury and acquired hypogonadism. This can be ameliorated with exogenous testosterone supplementation.

7. Cass AS, and Luxenberg M (June 1991) Testicular Injuries. Urology, 37(6):528\{530. doi:10.1016/0090-4295(91)80317-z.

8. Lysiak, J, Nguygen Q, Kirby J, et al (2003) Ischemia-reperfusion of the murine testis stimulates the expression of proinflammatory cytokines and activation of c-jun N-terminal kinase in a pathway to E-selectin expression. Biology of Reproduction, 69(1):202-210. Doi:10.1095/biolreprod.102.013318.

9. Cross, J, Berman L, Elliott P, et al (1999) Scrotal trauma: a cause of testicular atrophy. Clinical Radiology, 54(5):317-320.

10. Redmond, EJ, MacNamara, FT, Giri, SK, et al (2018) Blunt testicular trauma - is surgical exploration necessary? Ir J Med Sci, 187: 1109. https://doi.org/10.1007/s11845-017-1724-7 